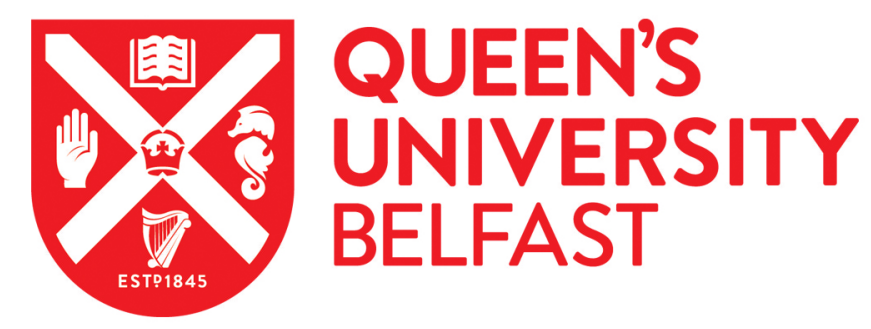

\title{
Patients with mesenchymal tumours and high Fusobacteriales prevalence have worse prognosis in colorectal cancer (CRC)
}

Salvucci, M., Crawford, N., Stott, K., Bullman, S., Longley, D. B., \& Prehn, J. H. M. (2021). Patients with mesenchymal tumours and high Fusobacteriales prevalence have worse prognosis in colorectal cancer (CRC). Gut. https://doi.org/10.1136/gutjnl-2021-325193

\section{Published in:}

Gut

Document Version:

Publisher's PDF, also known as Version of record

Queen's University Belfast - Research Portal:

Link to publication record in Queen's University Belfast Research Portal

\section{Publisher rights}

Copyright 2021 the authors. Published by BMJ.

This is an open access Creative Commons Attribution-NonCommercial License (https://creativecommons.org/licenses/by-nc/4.0/), which permits use, distribution and reproduction for non-commercial purposes, provided the author and source are cited

\section{General rights}

Copyright for the publications made accessible via the Queen's University Belfast Research Portal is retained by the author(s) and / or other copyright owners and it is a condition of accessing these publications that users recognise and abide by the legal requirements associated with these rights.

Take down policy

The Research Portal is Queen's institutional repository that provides access to Queen's research output. Every effort has been made to ensure that content in the Research Portal does not infringe any person's rights, or applicable UK laws. If you discover content in the Research Portal that you believe breaches copyright or violates any law, please contact openaccess@qub.ac.uk. 


\title{
(2) OPEN ACCESS
}

\section{Patients with mesenchymal tumours and high Fusobacteriales prevalence have worse prognosis in colorectal cancer (CRC)}

\author{
Manuela Salvucci (1), ${ }^{1}$ Nyree Crawford, ${ }^{2}$ Katie Stott, ${ }^{2}$ Susan Bullman, ${ }^{3}$ \\ Daniel B Longley, ${ }^{2}$ Jochen H M Prehn ${ }^{1}$
}

- Additional supplemental material is published online only. To view, please visit the journal online (http://dx.doi.org/ 10.1136/gutjnl-2021-325193).

${ }^{1}$ Centre for Systems Medicine, Department of Physiology and Medical Physics, Royal College of Surgeons in Ireland, Dublin, Ireland

${ }^{2}$ Patrick G Johnston Centre for Cancer Research, School of Medicine, Dentistry and Biomedical Science, Queen's University Belfast, Belfast, UK ${ }^{3}$ Human Biology, Fred Hutchinson Cancer Research Center, Seattle, Washington, USA

\section{Correspondence to}

Professor Jochen H M Prehn, Department of Physiology and Medical Physics and Centre for Systems Medicine, Royal College of Surgeons in Ireland, Dublin, Ireland; jprehn@rcsi.ie

Received 18 May 2021 Accepted 31 August 2021

Check for updates

(c) Author(s) (or their employer(s)) 2021. Re-use permitted under CC BY-NC. No commercial re-use. See rights and permissions. Published by BMJ.

\footnotetext{
To cite: Salvucci M, Crawford N, Stott K, et al. Gut Epub ahead of print: [please include Day Month Year]. doi:10.1136/ gutjnl-2021-325193
}

\begin{abstract}
Objectives Transcriptomic-based subtyping, consensus molecular subtyping (CMS) and colorectal cancer intrinsic subtyping (CRIS) identify a patient subpopulation with mesenchymal traits (CMS4/CRIS-B) and poorer outcome. Here, we investigated the relationship between prevalence of Fusobacterium nucleatum (Fn) and Fusobacteriales, CMS/ CRIS subtyping, cell type composition, immune infiltrates and host contexture to refine patient stratification and to identify druggable context-specific vulnerabilities.

Design We coupled cell culture experiments with characterisation of Fn/Fusobacteriales prevalence and host biology/microenviroment in tumours from two independent colorectal cancer patient cohorts (Taxonomy: $n=140$, colon and rectal cases of The Cancer Genome Atlas (TCGA-COAD-READ) cohort: $n=605$ ). Results In vitro, Fn infection induced inflammation via nuclear factor kappa-light-chain-enhancer of activated B cells/tumour necrosis factor alpha in HCT116 and HT29 cancer cell lines. In patients, high Fn/Fusobacteriales were found in CMS1, microsatellite unstable () tumours, with infiltration of M1 macrophages, reduced M2 macrophages, and high interleukin (IL)-6//L-8/LL-1 $\beta$ signalling. Analysis of the Taxonomy cohort suggested that $F n$ was prognostic for CMS4/CRIS-B patients, despite having lower Fn load than CMS1 patients. In the TCGA-COAD-READ cohort, we likewise identified a differential association between Fusobacteriales relative abundance and outcome when stratifying patients in mesenchymal (either CMS4 and/or CRIS-B) versus non-mesenchymal (neither CMS4 nor CRIS-B).

\section{Significance of this study}

What is already known on this subject?

- Fusobacterium nucleatum (Fn), a commensal Gram-negative anaerobe from the Fusobacteriales order, is an oncobacterium in colorectal cancer (CRC), and a causal relationship between $F n$ prevalence and CRC pathogenesis, progression and treatment response has been reported in vivo.

- Broad-spectrum antibiotics have proven moderately successful in reducing tumour growth promoted by $F n$ in preclinical models. However, the use of antibiotics to treat bacterium-positive cases in the clinic is not a viable option as it may further alter the already dysbiotic gut microbiome of patients with CRC and may also have limited efficacy against $F n$, which penetrates and embeds deeply within the tumour.

- The highly heterogeneous population of patients with CRC can be classified into distinct molecular subtypes (consensus molecular subtyping (CMS) and colorectal cancer intrinsic subtyping (CRIS)) based on gene expression profiles mirroring the underlying transcriptional programmes. Patients classified as CMS4 and CRIS-B exhibit a mesenchymal phenotype and have poorer outcome.
\end{abstract} Patients with mesenchymal tumours and high Fusobacteriales had approximately twofold higher risk of worse outcome. These associations were null in non-mesenchymal patients. Modelling the three-way association between Fusobacteriales prevalence, molecular subtyping and host contexture with logistic models with an interaction term disentangled the pathogen-host signalling relationship and identified aberrations (including NOTCH, CSF1-3 and IL-6/IL-8) as candidate targets.

Conclusion This study identifies CMS4/CRIS-B patients with high Fn/Fusobacteriales prevalence as a highrisk subpopulation that may benefit from therapeutics targeting mesenchymal biology.

\section{INTRODUCTION}

Colorectal cancer (CRC) has one of the highest morbidities and mortality rates among solid cancers, and its incidence is steadily on the rise, accounting for circa $10 \%$ of newly diagnosed cancer cases worldwide. ${ }^{1}$ Patients with CRC with similar macroscopic clinicopathological characteristics exhibit a high degree of heterogeneity at the molecular level, which translates into heterogeneous and often suboptimal response to treatment. Thus, research has focused on molecular subtyping strategies based on single or multiomics data from the host to categorise patients into subgroups to aid in risk stratification and disease management. Subtyping strategies such as the consensus molecular subtyping $\left(\mathrm{CMS}^{2}\right)$ and the colorectal cancer intrinsic subtyping $\left(\mathrm{CRIS}^{3}\right)$ classify patients into subgroups with more homogeneous signalling features based on key transcriptomic programmes. Among the four subtypes identified by the CMS classifier, CMS4 patients have high stroma infiltration along with upregulated angiogenesis and transforming growth 
Significance of this study

What are the new findings?

- FnlFusobacteriales prevalence is associated with immune involvement (decrease in antitumour M1 macrophages and increase in protumour M2 macrophages) and activation of specific signalling programmes (inflammation, DNA damage, WNT, metastasis, proliferation and cell cycle) in the host-tumours.

- The prevalence of bacteria from the Fusobacteriales order, largely driven by $F n$ species, plays an active or opportunistic role, depending on the underlying host-tumour biology and microenvironment.

- Fn and other species of the Fusobacteriales order are enriched in CMS1 (immune-high, microsatellite unstable tumours) patients compared with CMS2-4 cases.

- FnlFusobacteriales prevalence is associated with worse clinical outcome in patients with mesenchymal-rich CMS4/ CRIS-B tumours but not in patients with other molecular subtypes.

\section{How might it impact on clinical practice in the foreseeable} future?

- FnlFusobacteriales screening and transcriptomic-based molecular subtyping should be considered to identify patients with mesenchymal-rich tumours and high bacterium prevalence to inform disease management.

- Fn/Fusobacteriales prevalence may need to be addressed exclusively in patients with mesenchymal-rich high-stromal infiltrating tumours rather than a blanket approach to treat all pathogen-positive patients.

- Clinical management of the disease for this subpopulation of high-risk patients with unfavourable clinical outcome could be attained by administering agents currently in clinical trials that target aberrations in the host signalling pathways (NOTCH, WNT and epithelial-mesenchymal transition) and tumour microenviroment (inflammasome, activated T cells, complement system, and macrophage chemotaxis and activation).

factor- $\beta$ (TGF- $\beta$ ) signalling and show poorer recurrence-free and overall survival. ${ }^{2}$ Similarly, CRIS-B patients feature mesenchymal traits and also exhibit poorer outcome compared with patients classified as CRIS-A and CRIS-C-E. ${ }^{3}$

Recent research has identified the microbiome as a key player in health and disease, including cancer. ${ }^{4}$ Several research groups, including ours, have shown that Fusobacteriales, largely from Fusobacterium nucleatum (Fn), are more abundant in tumour tissue compared with matched adjacent mucosa,${ }^{5}$ suggesting a causative role in CRC progression. ${ }^{6}$ More advanced, right-sided, MSI tumours are typically enriched with $\mathrm{Fn}^{7}{ }^{7}$ Remarkably, antimicrobial treatment has been shown to reduce tumour burden in mouse xenograft models, ${ }^{8}$ corroborating the association between Fn-positive patients and poorer outcome observed in some studies. ${ }^{5}$ However, the prognostic value of $F n$ prevalence was not observed in other cohort studies (reviewed in GethingsBehncke et $a l^{9}$ ). Thus, we hypothesised that the impact of $F n / F u$ sobacteriales may differ according to the underlying tumour biology.

In this study, we combined mechanistic in vitro experiments in colon cancer cells with an in-depth analysis in two independent CRC patient cohorts and a systematic multiomic characterisation of cell signalling and tumour microenvironment in $\mathrm{n}=745$ patients to investigate the interaction between the dysregulation induced by Fusobacteriales prevalence (including $F n$ ) on the human host and, conversely, the characteristics of the host microenvironment that allow pathogens to thrive. Here, we provide evidence that the prognostic value of Fn/Fusobacteriales strongly relates to the molecular subtype of the host-tumour and is confined to subtypes showing mesenchymal involvement.

\section{MATERIALS AND METHODS}

Detailed methods for the in vitro experiments and the patients' study (design, cohorts' description and analysis steps) are provided in the online supplemental materials and methods.

\section{RESULTS \\ $F n$ infection induces inflammation mediated by tumour necrosis factor alpha (TNF- $\alpha$ ) and NFKB in CRC cellular cultures}

Due to the presence of $F n$ in CRC tumour tissue, ${ }^{58}$ a causative role for this bacterium in exacerbating tumourigenesis has been put forward. Infection of colon cells with $F n$ has previously been shown to induce inflammation, activate NFkB signalling and increase expression of the proinflammatory cytokine TNF- $\alpha^{10}{ }^{11}$ (figure 1A). Hence, we infected HCT116 and HT29 colon cancer cell line cultures for 6 hours to assess epithelial cell response to increasing amounts of $F n$ (multiplicity of infection, (MOI), bacteria-to-cancer-cells: 10, 100 and 1000). We found that NFKB signalling was activated on infection with $F n$ in CRC cell lines, as evidenced by the degradation of IKB $\alpha$ (alpha nuclear factor of kappa light polypeptide gene enhancer in B-cell inhibitor) (figure 1B), an increase in NFKB transcriptional activity (figure $1 \mathrm{C}$ ) and a marked increase in mRNA expression of the NFKB target gene, TNF- $\alpha$ (figure 1D). Taken together, these results confirm that $F n$ coculture with human colon cancer epithelial cells promotes a proinflammatory response.

\section{Prevalence of FnIFusobacteriales in tumour resections}

We sought to investigate the relationship between inflammation in the human host and prevalence of Fn and Fusobacteriales in tumour resections of patients with CRC. We selected an in-house multicentre stage II-III cohort (Taxonomy, $\mathrm{n}=140^{12}{ }^{13}$ ) and the colon and rectal cases of The Cancer Genome Atlas (TCGACOAD-READ cohort, $\mathrm{n}=605$ patients; figure $2 \mathrm{~A}$ ) to encompass the heterogeneity of the CRC clinicopathological characteristics observed in the clinic. Demographic, clinicopathological characteristics for the Taxonomy and TCGA-COAD-READ cohorts are summarised in online supplemental table 1 . We determined Fn load by a targeted quantitative real-time PCR in tumour resections of the Taxonomy cohort where we detected $F n$ in $\mathrm{n}=101$ of $140(72 \%)$ patients (figure 2B and online supplemental table 2). The distribution of $F n$ positivity levels (relative to the human PGT gene) was heterogeneous, and we categorised patients as $F n$-high or $F n$-low using the $75^{\text {th }}$ percentile as cut-off (figure 2B). We estimated Fusobacteriales relative abundance (RA) in the TCGA-COAD-READ cohort from RNA sequencing data by mapping non-human reads to microbial reference databases and retaining only high-quality matches (see the Materials and methods section) with a PathSeq analysis ${ }^{14} 15$ (figure 2A and online supplemental table 2). For downstream analyses, we reported the RA at the order, family, genus and species taxonomic rank, and expressed it as percentage of the 
A
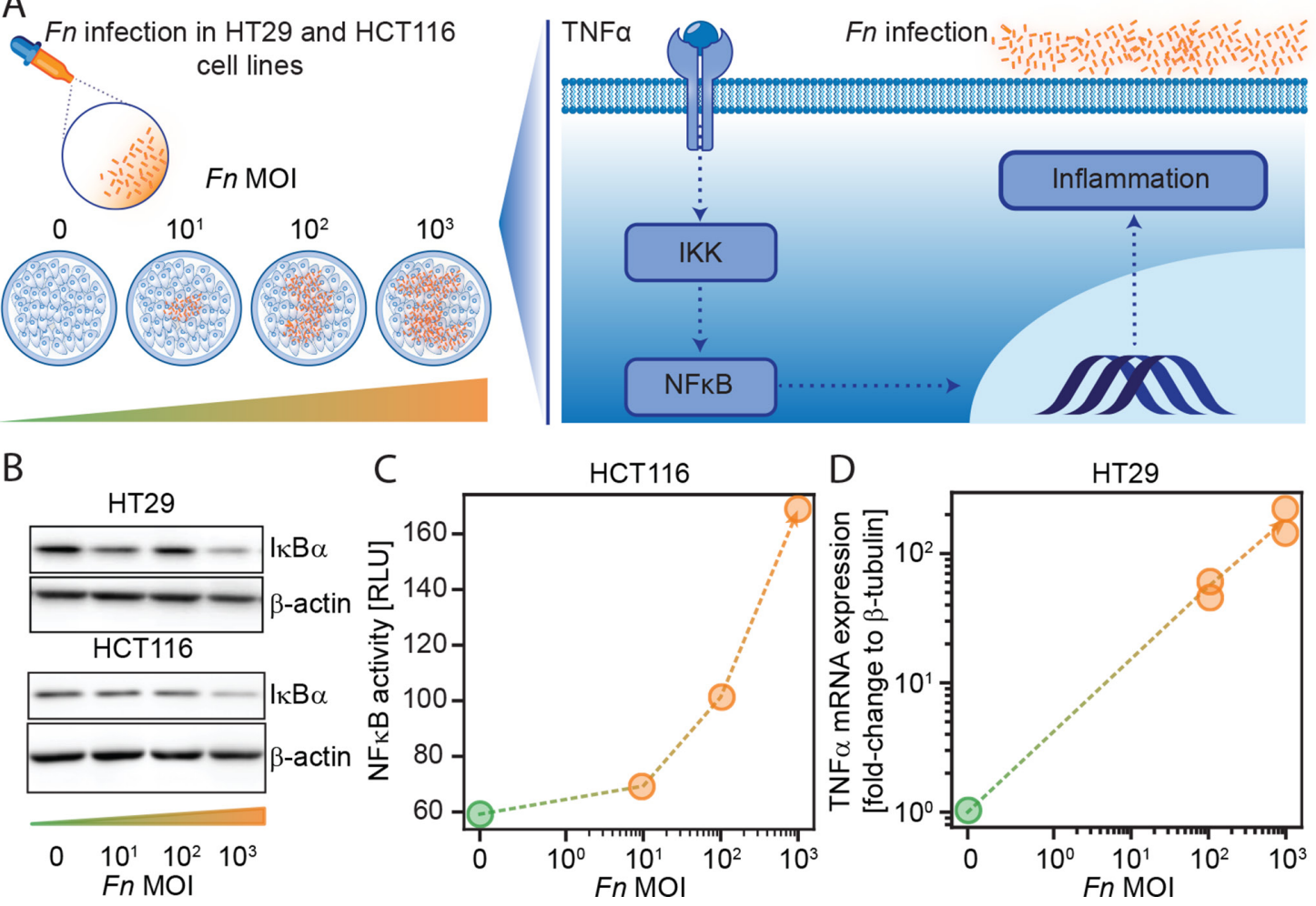

Figure 1 Fn infection induces inflammation mediated by TNF- $\alpha$ and NFKB in HCT116 and HT29 CRC cell lines. (A) Schematic representation of

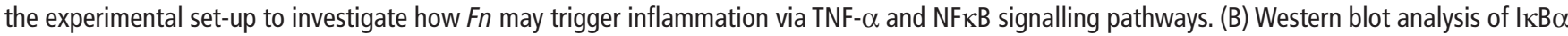
and $\beta$-actin in HT29 and HCT116 cell cultures following infection with Fn (MOI bacteria-to-cancer-cells 10, 100 and 1000). (C) NFkB transcriptional activity assay in HCT116 cells 6 hours following infection with Fn (MOI bacteria-to-cancer-cells 100 and 1000). (D) TNF- $\alpha$ mRNA expression relative to $\beta$-tubulin in HT29 cells 6 hours following infection with Fn (MOI bacteria-to-cancer-cells 100 and 1000). (B-D) Representative results from duplicate experiments. Fn, Fusobacterium nucleatum; TNF- $\alpha$, tumour necrosis factor alpha.

total bacterial abundance. We detected Fusobacteriales (defined as RA over zero, at the order level) in $n=558$ of 605 (92\%) of the TCGA-COAD-READ patients (figure 2D). Fn was the most abundant species and was detected in $82 \%$ of the TCGA-COADREAD patients (compared with $72 \%$ in the Taxonomy cohort), accounting on average for approximately $45 \%$ of total Fusobacteriales RA and accounting for over $75 \%$ of total Fusobacteriales $\mathrm{RA}$ in $16 \%$ of cases (figure 2C). Analogous to the Taxonomy cohort, we categorised patients as Fusobacteriales-high or Fusobacteriales-low using the $75^{\text {th }}$ percentile as cut-off.

\section{Higher FnIFusobacteriales prevalence correlates with inflammation and immune involvement}

We examined the association between host gene expression profiles of key inflammatory markers and either Fn load or Fusobacteriales RA in the Taxonomy and TCGA-COAD-READ cohorts, respectively. In line with the in vitro experiments (figure 1), we detected an increase in NFKB1 and a trend in TNF- $\alpha$ gene expression, recapitulated by transcriptomic-based signatures for an overall inflammation status mediated by the cytolytic and interferon gamma (IFN- $\gamma$ ) pathways in the Taxonomy cohort (figure 2E). When investigating further key inflammation players, we observed a marked increase in proinflammatory interleukins (ILs) (IL-6, IL-8, IL-10, IL-1 $\beta$ and IL-13), cytokines/chemokines (CCL8, CSF1 and ICAM1), metalloproteins (MMP1, MMP3 and MMP9), NOS2, the inflammasome complex (NLRP3) and decrease in COX2 in Fn-high versus $F n$-low Taxonomy patients (figure $2 \mathrm{E}$ and online supplemental figure 1).
We sought to validate and build on our findings from the in-house Taxonomy cohort by analysing the TCGA-COADREAD cohort (figure 2F). At the transcription level, we confirmed an exacerbated inflammatory state when comparing Fusobacteriales-high and Fusobacteriales-low patients mediated by the NFкB-TNF- $\alpha$ axis and IFN- $\gamma$ with cytolytic involvement. Fusobacteriales-high patients overexpressed proinflammatory ILs (IL-6, IL-8, IL-10 and IL-1 $\beta$ ), cytokines/chemokines (CCL8 and ICAM1), metalloproteinases (MMP1 and MMP3), NOS2 and inflammasome markers (NLRP3) (figure 2F).

As inflammation is strongly tied to immune cell migration and activity, we investigated whether there was a link between immune cell composition and either Fn load (taxonomy) or Fusobacteriales RA (TCGA-COAD-READ). Cell composition was computationally deconvoluted from gene expression profiles with quanTiseq ${ }^{16}$ and microenvironment cell populations (MCP)-counter ${ }^{17}$ (figure 2G,H). Despite observing high interpatient heterogeneity in cell composition within the Taxonomy and TCGA-COAD-READ cohorts, we detected higher immune cell activation and polarisation when comparing patients with high versus low Fn load (Taxonomy) or Fusobacteriales RA (TCGACOAD-READ). Patients with high Fn load (Taxonomy) or Fusobacteriales (TCGA-COAD-READ) showed higher predicted abundance of regulatory $\mathrm{T}$ cells coupled with an increase in M1 macrophages and a decrease in M2 macrophages (figure 2I,J). MCP-counter identified a strong positive association between neutrophil infiltration and either Fn load (Taxonomy) or Fusobacteriales RA (TCGA-COAD-READ). However, no difference in predicted neutrophils abundance was detected by quanTIseq. 

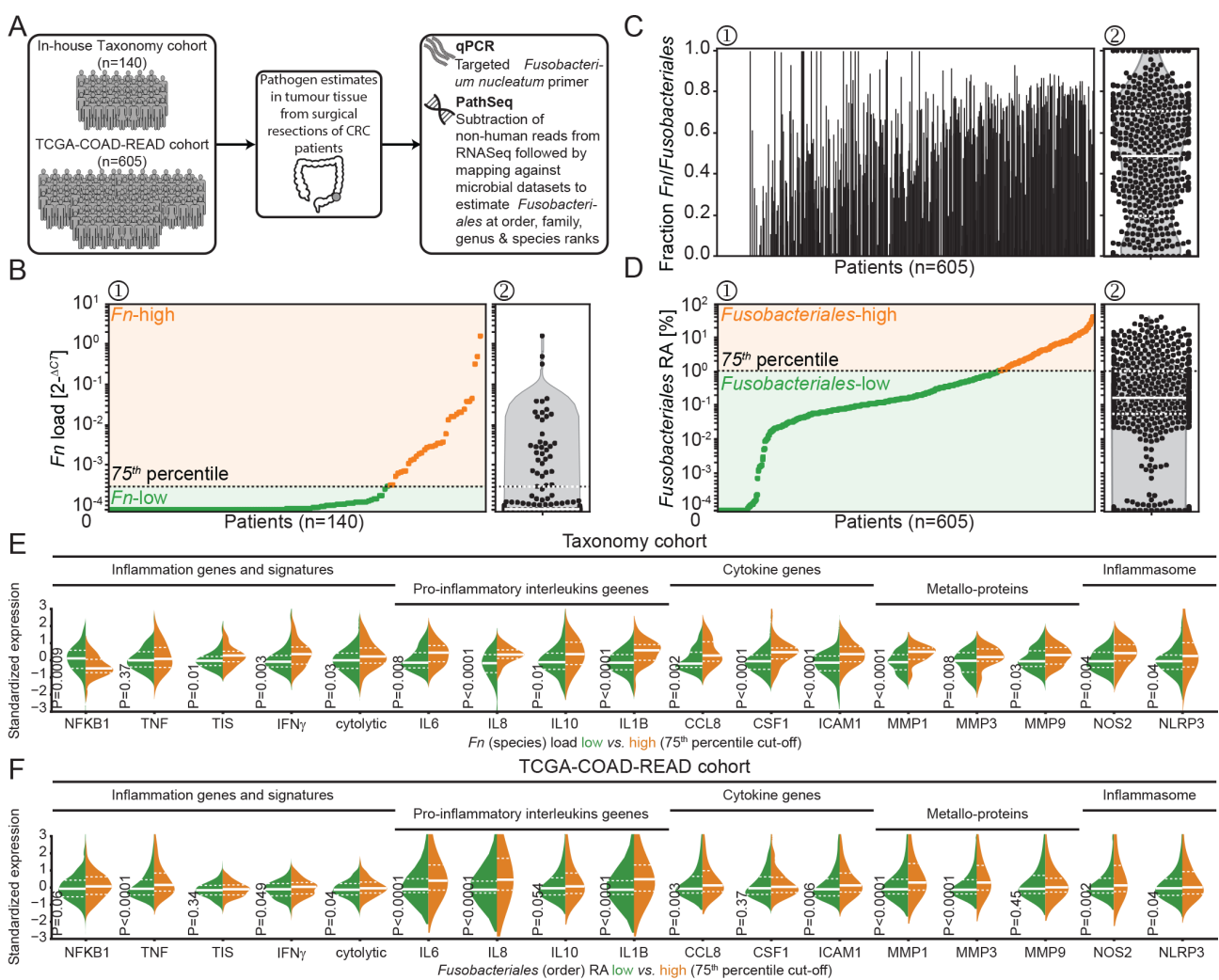

G

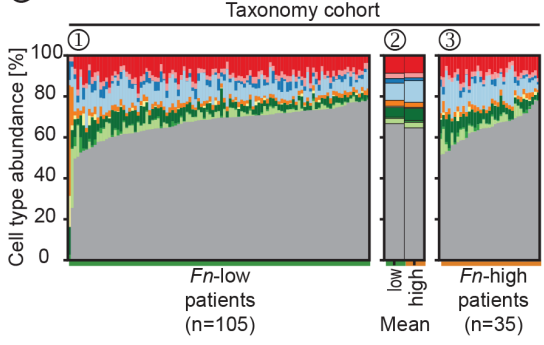

I

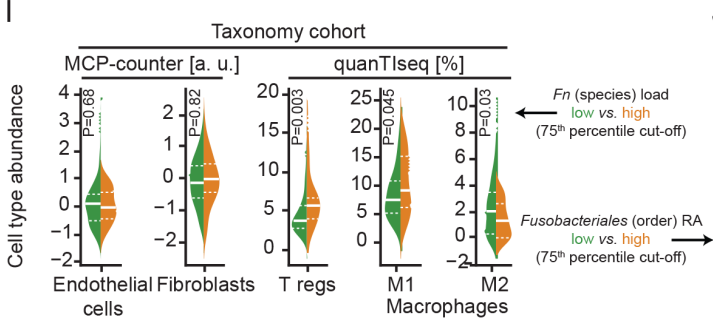

$\mathrm{H}$

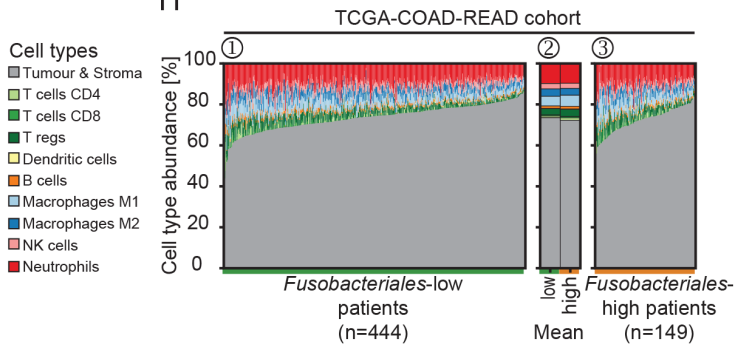

J

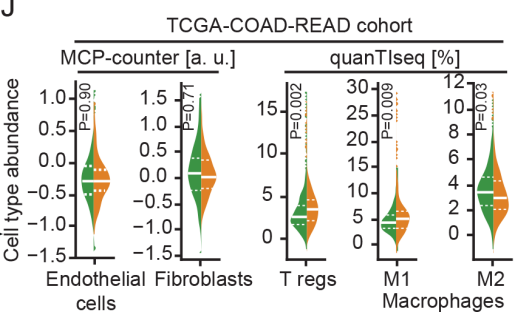

Figure 2 Fn/Fusobacteriales prevalence is associated with inflammation and immunosuppression in patients with CRC of the Taxonomy and TCGACOAD-READ CRC cohorts. (A) Schematic representation of the cohorts included in the study and methods to estimate Fn load and Fusobacteriales (order) RA in the Taxonomy and TCGA-COAD-READ cohorts, respectively. (B-D) Per-patient (waterfall plot, 1, left) and distribution (violin plot with overlaid data-points, 2 , right) of bacterium prevalence in tumour resections of the Taxonomy $(n=140, B)$ and TCGA-COAD-READ ( $n=605, D)$. In B,D 1, patients are sorted in ascending order of either Fn load (Taxonomy cohort, B) or Fusobacteriales RA at the order taxonomic rank (TCGA-COAD-READ cohort, D). Cut-off of $75^{\text {th }}$ percentile used for patients' stratification in downstream analysis is also indicated (black dotted line). (C) Corresponding per-patient fraction of $F n$ species to total Fusobacteriales order RA detected for the TCGA-COAD-READ cohort . (E,F) Violin plots depicting the expression distribution of key genes or signatures involved in inflammation and immunosuppression grouped by patients with low (in green) or high (in orange) either Fn load (Taxonomy cohort, E) or Fusobacteriales RA at the order taxonomic rank (TCGA-COAD-READ cohort, F). Median and lower $\left(25^{\text {th }}\right)$ and upper $\left(75^{\text {th }}\right)$ percentiles are indicated by white solid or dashed lines, respectively. Statistical significance was evaluated using Kruskal-Wallis tests and $p$ values are reported. $(G, H)$ Stacked bar plots indicating cell type composition per patient estimated from gene expression by quanTlseq in tumours with low versus high either Fn load (Taxonomy cohort, G) or Fusobacteriales RA at the order taxonomic rank (TCGA-COAD-cohort, H). Cell type composition is shown sorted in ascending order of tumour and stromal content (1 and 3) and aggregated (by mean, 2 across the low and high subgroups). (I,J) Distribution of specific tumour/stroma and immune cell types determined as indicated by either quanTIseq or MCP-counter grouped by either Fn load (Taxonomy cohort, I) or Fusobacteriales RA at the order taxonomic rank (TCGA-COAD-READ cohort, J). Median and lower (25 $5^{\text {th }}$ ) and upper $\left(75^{\text {th }}\right)$ percentiles are indicated by white solid or dashed lines, respectively. Statistical significance was evaluated using Kruskal-Wallis tests and p values are reported. CRC, colorectal cancer; Fn, Fusobacterium nucleatum; NK, natural killer cells; RA, relative abundance; TCGA-COAD-READ, colon and rectal cases of The Cancer Genome Atlas; Treg, regulatory T cell. 
Importantly, no difference in fibroblasts and endothelial cells

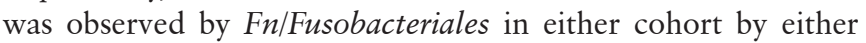
method (figure 2I,J).

\section{Multiomic characterisation of the association between Fusobacteriales RA and human host-tumour microenvironment in the TCGA-COAD-READ cohort}

We leveraged the rich molecular characterisation of the TCGACOAD-READ cohort to perform a systematic and unbiased characterisation of the association between Fusobacteriales RA and patient clinical and molecular features to identify human host vulnerabilities that may be conducive for tumour development (figure 3).

We observed higher Fusobacteriales in patients of older age, diagnosed with more advanced disease stage and tumours located in the colon, particularly in proximal site (figure 3A) cohorts. In contrast, we found no statistically significant differences in Fusobacteriales RA by sex, body mass index and either lymphovascular or perineural invasion (online supplemental figure 2). We observed similar patterns and a slightly higher prevalence in women (Taxonomy cohort, $\mathrm{p}=0.049$ ), when assessing $F n$ in both the TCGA-COAD-READ and Taxonomy cohorts (online supplemental figure 3A), corroborating previous studies. ${ }^{18}$

Patients harbouring higher Fusobacteriales showed lower genomic intratumour heterogeneity, had higher silent and non-silent mutational burden and were enriched in microsatellite unstable cases (figure 3B and online supplemental figure 3B). Fusobacteriales-high patients had an increase in transitions, defined as the exchange of two-ring purines $(A \leftrightarrow G)$ or of a one-ring pyrimidines $(C \leftrightarrow T)$, coupled with a decrease in transversions, a substitution of purine for pyrimidine bases (online supplemental figure $4 \mathrm{~A}$ ) as evidenced by a decrease in conversion changes of $\mathrm{C}>\mathrm{G}$ and $\mathrm{T}>\mathrm{A}$ (online supplemental figure 4B). We found no difference in prevalence of common mutations in CRC by Fusobacteriales (low vs high) except for $B R A F$ (figure 3C). BRAF mutations trended to be more common among Fusobacteriales-high and $F n$-high patients, as previously reported when assessing $\mathrm{Fn}^{18}$ (figure $3 \mathrm{C}$ and online supplemental figure 3B). A comprehensive screen revealed that mutations in cell cycle (ATM), Hedgehog signalling (MEGF8), DNA damage/repair (TRIP12 and PRKDC), mitotic spindle (ASPM) and migration/adhesion (TRIO, GPR98) were more prevalent in Fusobacteriales-high patients (figure 3D) (online supplemental table 3).

We set out to investigate the relationship between copy number alterations (CNAs) and Fusobacteriales presence in the TCGACOAD-READ cohort (figure 3E-G). We determined recurrent CNA amplifications and deletions across the whole cohort by applying the genomic identification of significant targets in cancer (GISTIC) algorithm ${ }^{19}$ (online supplemental figures 5 and 6 and online supplemental table 4). Fusobacteriales-high cases showed lower chromosomal instability with a lower fraction of the genome affected by recurrent CNAs, in line with the increased incidence of MSI. We identified CNA amplifications or deletions, the frequency of occurrence of which differed between Fusobacteriales-high versus Fusobacteriales-low patients and, thus, may be specifically associated with the bacterium presence (figure 3F). CNAs more frequently (>15\%) observed in Fusobacteriales-high versus Fusobacteriales-low cases included deletions in 8p23.2 (tumour suppressor CSMD1 and LOC100287015), 18q21.1 (MIR4743 and RNA binding by CTIF) and 18q23, which impact the regulation of IL-6 and chemokine secretion, cell-cell adhesion and host response of viral transcription (figure $3 \mathrm{G}$ ).

We then focused on the transcriptional level and combined enrichment analyses with pathway-activity signatures to compare the impact of Fusobacteriales RA on cellular processes (figure $3 \mathrm{H}-\mathrm{J}$ ). Transcriptional profiles that differed included mTORC1 and cMYC signalling, cell cycle (G2-M checkpoint), mitotic spindle, epithelial-to-mesenchymal transition, TGF- $\beta$ and IL-1 regulation of extracellular matrix, matrix remodelling including focal adhesion, cytoskeleton and contractile actin filament bundle, mitochondrial translational elongation/termination, and protein complex assembly and stromal estimates (figure $3 \mathrm{H}$,I, online supplemental figure 7 and supplemental table 5 ). We corroborated these findings by comparing the activation of signalling pathways estimated by gene set signatures identified in the literature (see the Materials and methods section) in Fusobacteriales-low versus Fusobacteriales-high patients. Fusobacteriales RA was inversely linked to WNT signalling and positively associated with proliferation, metastasis (figure 3J) and DNA damage.

We sought to investigate whether the findings at the genomic and transcriptional levels were also observed in protein profiles determined by reverse phase protein array. We found that Fusobacteriales RA correlated with differential expression of proteins involved in microenvironment composition (Claudin7), cell cycle (Cyclin1), cMYC, apoptosis (cleaved Caspase7), proliferation (DLV3), Hippo pathway (Yap), DNA damage (Chk1 and ATM), receptor and mitogen-activated protein(MAP) kinases and PI3K signalling (figure $3 \mathrm{~K}-\mathrm{M}$, online supplemental figure 8 and supplemental table 6).

\section{FnIFusobacteriales prevalence differs by transcriptomic-based molecular subtype}

The aforementioned systematic screen pinpointed host aberrations associated with Fusobacteriales hallmarked by transcriptomics-based molecular subtypes. Hence, we classified patients in the study by $\mathrm{CMS}^{2}$ and CRIS ${ }^{3}$ subtyping. We observed higher Fn load (Taxonomy, figure 4A) and Fusobacteriales RA (TCGA-COAD-READ, figure 4C) in immune-high CMS1 tumours, corroborating the link between pathogen prevalence and host immunity. We observed higher Fn load in CRIS-B tumours (figure 4B) and Fusobacteriales RA in CRIS-A cases (figure 4D) of the Taxonomy and TCGA-COAD-READ cohorts, respectively. At the family rank, Fusobacteriaceae were more abundant than Leptotrichiaceae, accounting for 77\% and 23\% of total Fusobacteriales RA and $\sim 2 \%$ and $\sim<1 \%$ of the total bacteria RA, respectively. In line with the findings at the order level, we observed an increase in Fn, the most abundant Fusobacterium species, in CMS1 and CRIS-A cases (figure 4E,F). In line with the findings at the order level, we observed an approximately threefold increase when comparing patients classified as CMS1 versus the rest (figure 4E). Fn, the most abundant Fusobacterium species, was enriched in CMS1 and CRIS-A cases (figure $4 \mathrm{E}, \mathrm{F}$ ). We examined whether the positive association between inflammation and immune involvement by Fn/Fusobacteriales presence could be ascribed to the host CMS1 milieu or whether there was an additional pathogen-induced component. When restricting the analysis to CMS1 cases, we observed higher expression of proinflammatory markers in Fusobacteriales-high patients of the TCGA-COAD-READ cohort. We detected no association between pathogen prevalence and expression of antiinflammatory markers or inflammation signatures in either CRC cohort (figure 4G,H). Taken together, these results suggest that 

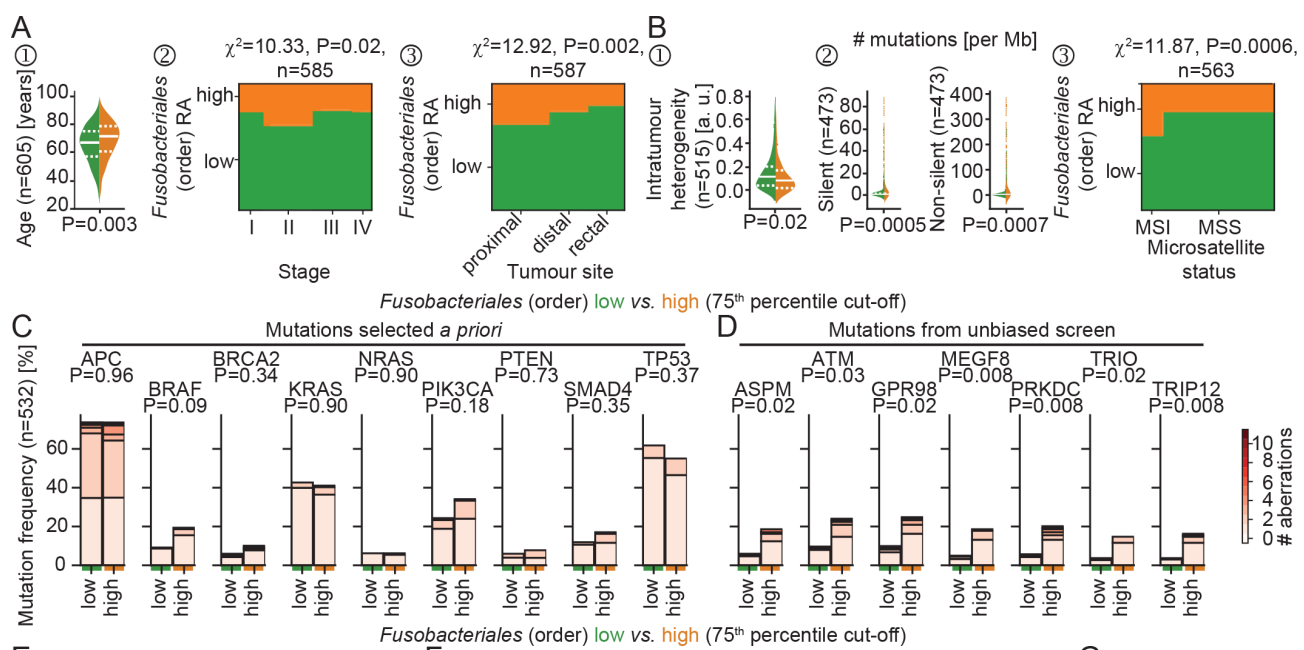

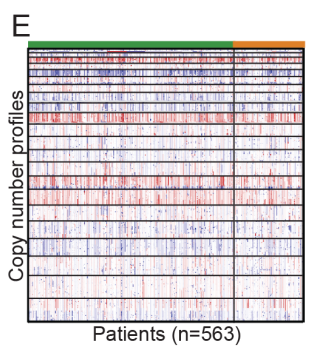

$\mathrm{H}$
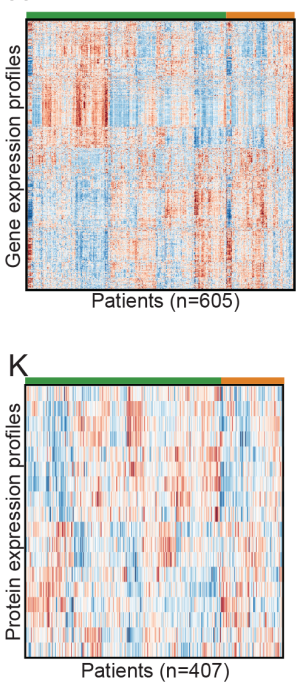

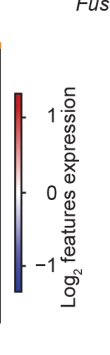

$\mathrm{F}$ 。

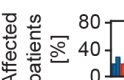

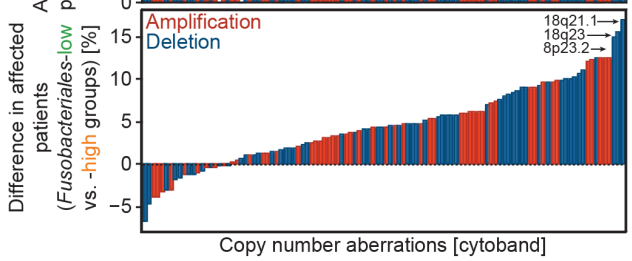

I
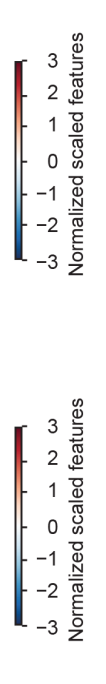

要

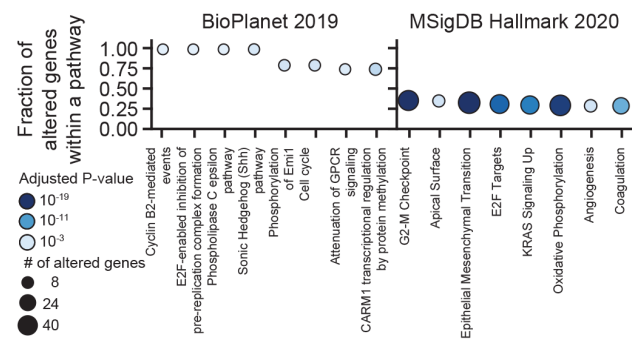

$\mathrm{L}$

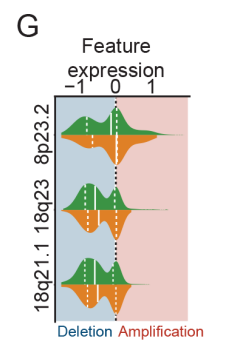

$J$

Standardized expression

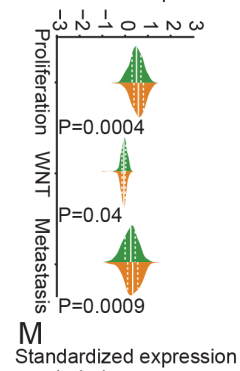

Standardized expression

$\dot{\omega} \omega^{\prime} O-N \omega$

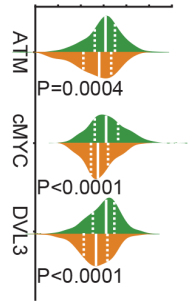

Figure 3 Multiomic characterisation of the association between Fusobacteriales RA and human host-tumour microenvironment in the TCGACOAD-READ cohort. $(A, B)$. Association between Fusobacteriales at the order taxonomic rank binned into low versus high (cut-off $75^{\text {th }}$ percentile) and clinicopathological (A) and mutational (B) characteristics of the human host. (C,D) Comparison of frequency of occurrence of mutations selected a priori (C) or identified by an unbiased scan (D) in Fusobacteriales-low versus Fusobacteriales-high patients. Colour bar indicates number of detected aberrations among frame shift deletions and insertions, in frame deletions and insertions, missense and nonsense mutations, and splice sites. P values were computed with $\chi^{2}$ independence tests and adjusted for multiple comparisons (Benjamini-Hochberg false discovery rate). (E-G) Heatmap (E) displaying copy number alterations grouped by Fusobacteriales-low (in green) and Fusobacteriales-high (in orange) RA. Waterfall plot (F) displaying differences in recurrent copy number aberrations detected in patients with low Fusobacteriales versus high Fusobacteriales. Top panel (F) reports percentage of patients affected by recurrent copy number aberrations. Distribution of top 3 deletions, the frequency of occurrence of which differs between Fusobacteriales-low and Fusobacteriales-high patients (G). Red and blue shadings indicate amplification and deletions, respectively. (H-J) Heatmap $(\mathrm{H})$ displaying expression of genes differentially expressed when comparing Fusobacteriales-low versus Fusobacteriales-high patients and corresponding pathway enrichment analysis (I). Expression distribution grouped by Fusobacteriales RA (low, in green, vs high, in orange) for selected gene expression signatures (J). (K-M) Heatmap (K) displaying expression of proteins differentially expressed when comparing Fusobacteriales-low versus Fusobacteriales-high patients and corresponding pathway enrichment analysis (L). Expression distribution grouped by Fusobacteriales RA (low, in green, vs high, in orange) for key proteins $(\mathrm{M})$. In violin plots, the median and lower $\left(25^{\text {th }}\right)$ and upper $\left(75^{\text {th }}\right)$ percentiles are indicated by white solid or dashed lines, respectively. Green and orange annotation bars denote patients with low versus high Fusobacteriales RA (75 ${ }^{\text {th }}$ percentile cut-off). (Unadjusted) P values (J,M) were determined by Kruskal-Wallis tests. MSS, patients with microsatellite stable tumours; RA, relative abundance; TCGACOAD-READ, colon and rectal cases of The Cancer Genome Atlas. 

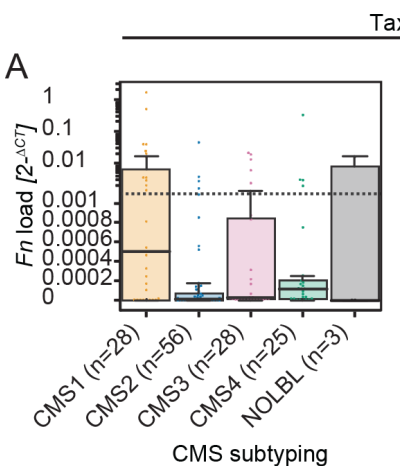

CMS subtyping

TCGA-COAD-READ cohort

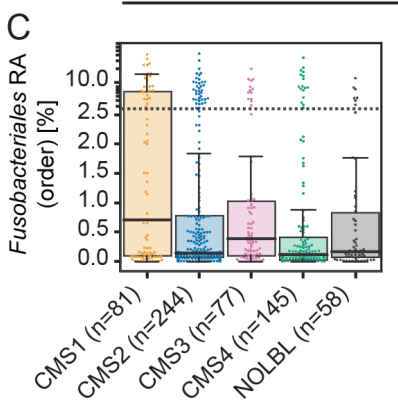

CMS subtyping

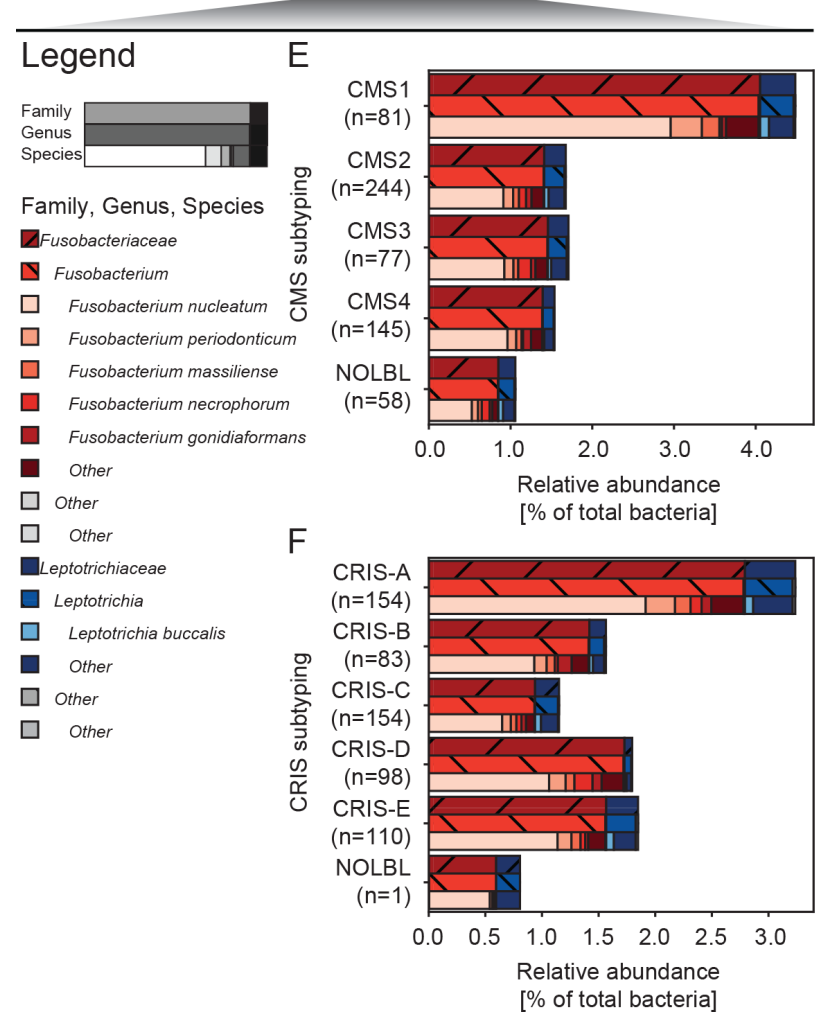

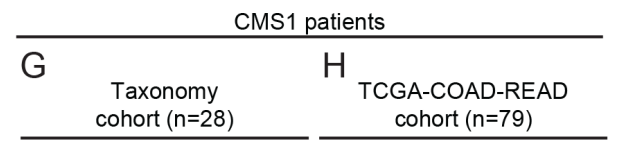

Key inflammatory gene expression
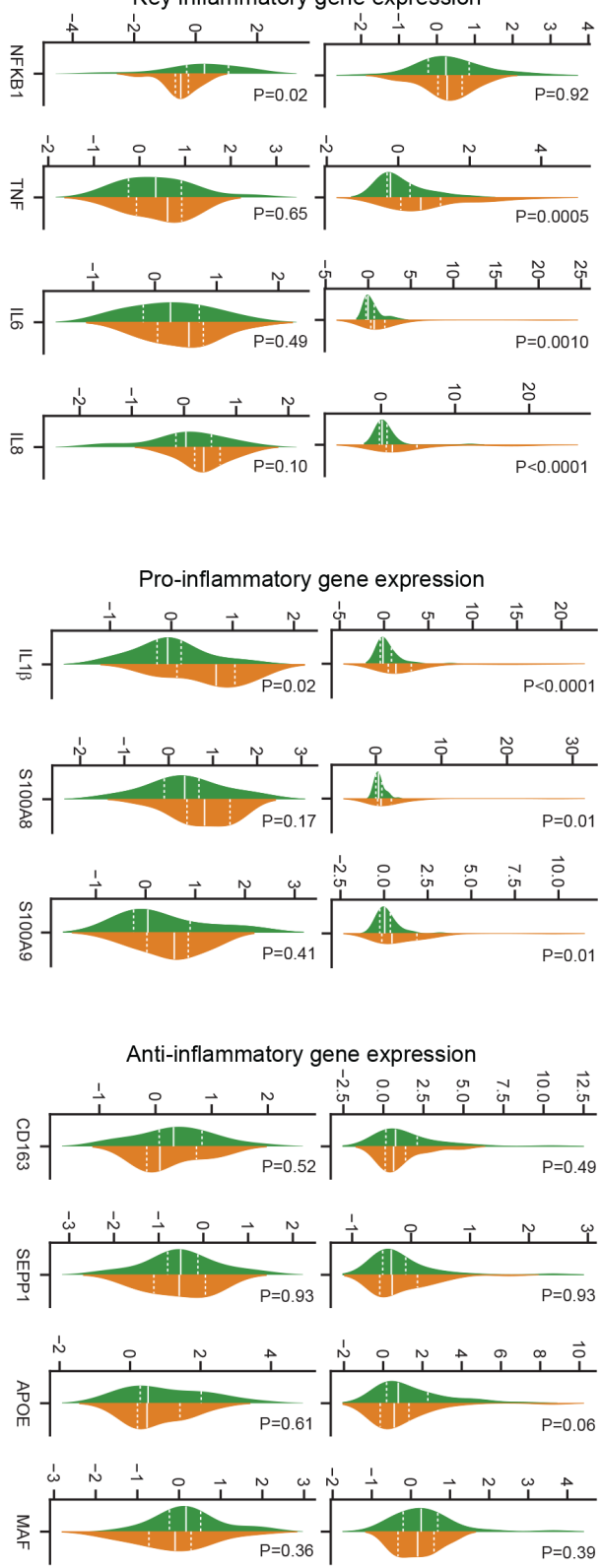

Fn (species) Fusobacteriales (order) low vs. high ( $75^{\text {th }}$ percentile cut-off)

Figure 4 Prevalence of Fn/Fusobacteriales by transcriptomic-based molecular subtypes of the host. (A-D) Boxplot with overlaid dot plots displaying the dependency by CMS $(A, C)$ and CRIS $(B, D)$ molecular subtyping by either Fn load (Taxonomy cohort; $A, B$ ) or Fusobacteriales RA at the order taxonomic rank (TCGA-COAD-READ cohort; $C, D)$. (E,F) RA (to total bacterial kingdom) of Fusobacteriales reported at increasing resolution of taxonomic rank (family, genus and species) by CMS (E) and CRIS (F) subtypes (aggregated by mean). Genuses/species with an average RA lower than 0.05 were aggregated as 'other'. (G,H) Distribution of key (pro-)/(anti-)inflammatory genes in CMS1 patients classified as -low (in green) or -high (in orange) using the $75^{\text {th }}$ percentile as cut-off. Patients' stratification was based on either Fn load (Taxonomy cohort (G) or Fusobacteriales RA at the order taxonomic rank (TCGA-COAD-READ cohort, H). Median and lower $\left(25^{\text {th }}\right)$ and upper $\left(75^{\text {th }}\right)$ percentiles are indicated by white solid or dashed lines, respectively. (Unadjusted) P values were determined by Kruskal-Wallis tests. CMS, consensus molecular subtyping; CRIS, colorectal cancer intrinsic subtyping; Fn, Fusobacterium nucleatum; RA, relative abundance; TCGA-COAD-READ, colon and rectal cases of The Cancer Genome Atlas. 
Fn/Fusobacteriales may play an active role in mediating inflammation in the host.

\section{Patients with high FnlFusobacteriales have worse outcome in CMS4/CRIS-B}

We sought to investigate whether bacterium presence correlated with patient clinical outcome assessed by overall survival (OS), disease-specific survival (DSS) and disease-free survival (DFS) endpoints (figure 5 and online supplemental figures 9 and 10).

We found no statistically significant differences in either cohort when comparing survival curves from patients grouped by either Fn load or Fusobacteriales RA (figure 5A,E,I and online supplemental figure 9-10). We hypothesised that Fn/Fusobacteriales may result in poorer outcome in a subtype-dependent context (ie, mesenchymal status; figure 5B,F,J). Indeed, we identified a differential association between Fusobacteriales RA and clinical outcome of the TCGA-COAD-READ cohort in mesenchymal (either CMS4 and/or CRIS-B) versus non-mesenchymal (neither CMS4 nor CRIS-B) tumours (figure 5 G,H,K,L and online supplemental figure 10). Fusobacteriales-high mesenchymal patients had approximately twofold higher risk of worse outcome, whereas these associations were null in non-mesenchymal patients (figure $5 \mathrm{G}, \mathrm{H}, \mathrm{K}, \mathrm{L}$ and online supplemental figure 10 ). Importantly, these findings held true when accounting for key (adjusted model 1) and more extensive (adjusted model 2) clinical-pathological characteristics that may represent confounders or disease modifiers (online supplemental table 7). We fitted two additional Cox regression models where, in addition to the interaction term between Fusobacteriales and mesenchymal status, we included adjustment covariates. In adjusted model 1, we included age, stage, tumour location and sex as key clinicopathological and demographic covariates. In adjusted model 2 , we expanded on adjusted model 1 by also including history of colon polyps and history of other malignancy as comorbidities. We found that the risk of unfavourable outcome (HRs) and statistical significance were minimally impacted by accounting for potential disease modifiers in adjusted models 1 and 2, confirming the robustness of our findings (online supplemental table 7).

Although numbers in the Taxonomy cohort are more limited, when restricting the analysis to CMS4 and/or CRIS-B cases, we observed a trend in which $F n$-high patients had shorter OS than those with low Fn load. Again, no difference in survival according to $F n$ load was observed in non-mesenchymal Taxonomy patients (figure 5C and online supplemental figure 9).

Exploratory analyses examining the association between clinical outcome and pathogen prevalence at taxonomic ranks of increasing resolution (order, family, genus and species) in the TCGA-COAD-READ cohort by fitting Cox regression models on the whole unselected population and in mesenchymal versus non-mesenchymal settings revealed that the prognostic impact stems primarily from, but is not limited to, species, including Fn, from the Fusobacterium genus from the Fusobacteriaceae family (figure $5 \mathrm{M}$ and online supplemental figure 11).

\section{Putative mechanisms underlying selective Fusobacteriales virulence in mesenchymal tumours}

Having identified a patient subpopulation that has an unfavourable clinical outcome when their tumours exhibit mesenchymal traits and are highly positive with Fn/Fusobacteriales, we reasoned that intervening by either clearing Fn/Fusobacteriales with broad-spectrum antibiotics or targeting the host-tumour biology could ameliorate clinical outcome for this subpopulation of patients. Given that broad-spectrum antibiotics may not represent a viable avenue in the clinic and narrow-spectrum antibiotics currently do not exist, we set out to identify clinically actionable host-specific vulnerabilities that could be exploited. We examined the host signalling pathways and microenvironment to identify alterations that may be mediated by and/or exacerbated by Fusobacteriales (ie, interact) and, thus, may promote virulence and, ultimately, result in an unfavourable clinical outcome. To disentangle the three-way association between Fusobacteriales RA, gene/signature and molecular subtyping, we fitted two distinct logistic regression models for each feature of interest in the TCGA-COAD-READ cohort. The selection of features was hypothesis-driven and included key host signalling pathways and immunomodulators (figure 6A).

Figure $6 \mathrm{~A}$ reports $\mathrm{p}$ values from the two models capturing the association between Fusobacteriales RA (high vs low) and either each gene/signature (model 1: Fusobacteriales gene/ signature, $\mathrm{x}$-axis) or the interaction between each gene/signature with the molecular subtype (model 2: Fusobacteriales gene) signature $\times$ molecular subtype, $\mathrm{y}$-axis). The top half quadrant (darker grey shaded area) identifies a set of genes/signatures whose expression patterns differ by molecular subtype (statistically significant interaction $p$ value in model 2) and thus may be mediating the signalling impact of Fusobacteriales and were prioritised for downstream analyses (figure 6B).

We tested whether the gene/signature we identified as candidate targets are indeed related to clinical outcome in patients of the TCGA-COAD-READ cohort with mesenchymal tumours and high Fusobacteriales. We restricted our analysis to patients with mesenchymal tumours, and for each clinical endpoint of interest, namely, OS, DSS or DFS, we fitted Cox regression models with an interaction term for Fusobacteriales RA (low vs high) and each of the gene/signature (low vs high) identified as statistically significant in the analysis presented in figure 6A. We reasoned that a gene/signature could be considered a candidate target with both specific and translatable impact on clinical outcome for patients with mesenchymal tumours if its association with unfavourable clinical outcome differed by Fusobacteriales. This analysis identified CSF1-3, IL- $1 \beta$, IFN- $\gamma$, IL-8, IL-6, CD163, NOTCH2, ZEB2 and TFF2 as potential targets for patients with mesenchymal tumours and high Fusobacteriales (figure 6C and online supplemental figures 12-14).

\section{DISCUSSION}

Fusobacteriales, predominantly Fn, have been associated ${ }^{5681120-26}$ with pathogenesis, progression and treatment response in CRC. We coupled mechanistic studies in cell cultures with hypothesisdriven and unbiased screening in clinically relevant and omics-rich CRC cohorts to examine the cross-talk between pathogen-host and pathogen-tumour microenvironment. We demonstrate relationships between Fn/Fusobacteriales prevalence and host immunity, signalling and transcriptomic-based molecular subtypes. Our findings suggest that host-pathogen interactions can define patient subpopulations where Fn/Fusobacteriales play an active or opportunistic role, depending on the underlying host-tumour biology and microenvironment and identify putative druggable and clinically actionable vulnerabilities.

We observed higher Fn/Fusobacteriales prevalence in CMS1 patients, corroborating findings by Purcell et al. ${ }^{27}$ Interestingly, we found that overall, higher pathogen prevalence did not correlate with poorer disease outcome. In contrast, high $\mathrm{Fn} / \mathrm{Fu}$ sobacteriales levels were associated with poor prognosis in the CMS4/CRIS-B patient subset, suggesting that the presence of 


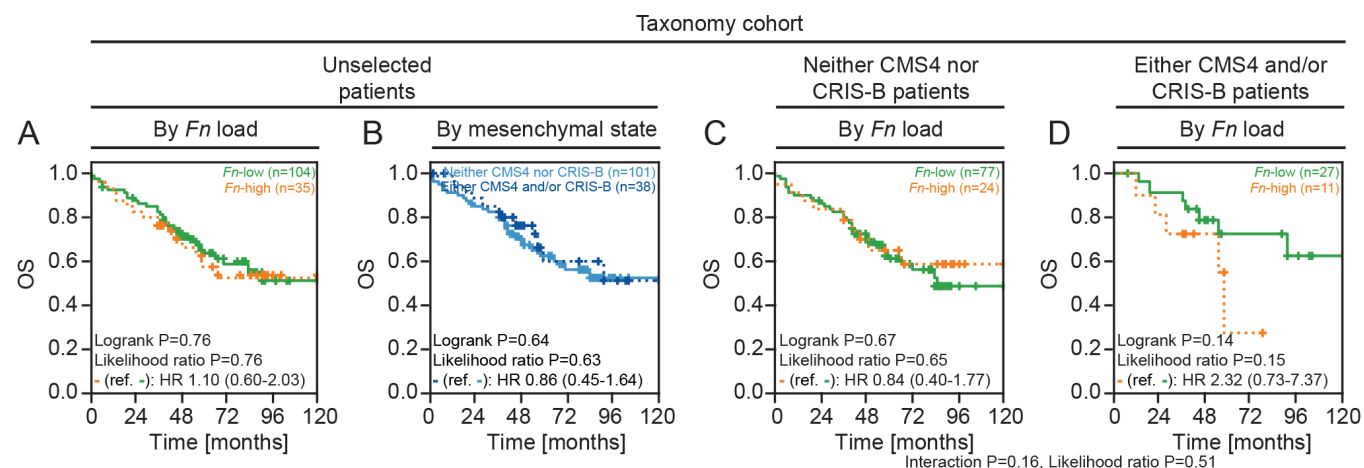

TCGA-COAD-READ cohort
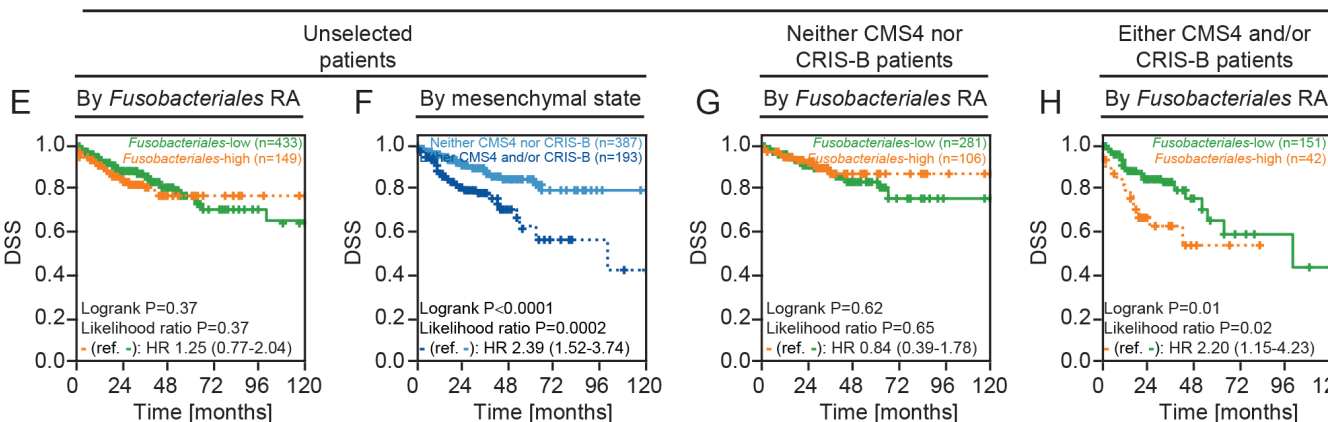

F By mesenchymal state

G By Fusobacteriales RA

H By Fusobacteriales RA
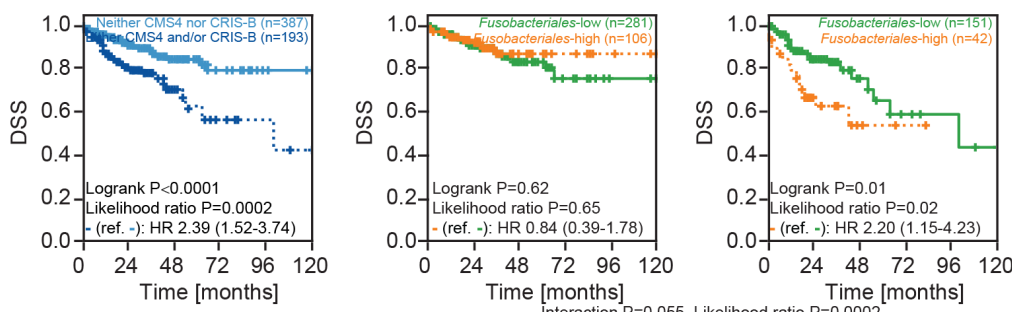
Time [months]

Neither CMS4 nor

Unselected

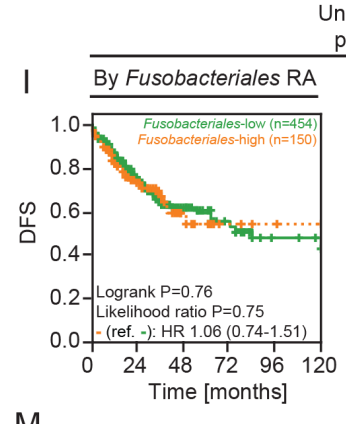

patients

CRIS-B patients

055 , Likelihood ratio $P=0.0002$ io $=0.0002$

K $\overline{\text { By Fusobacteriales RA }}$ CRIS-B patients
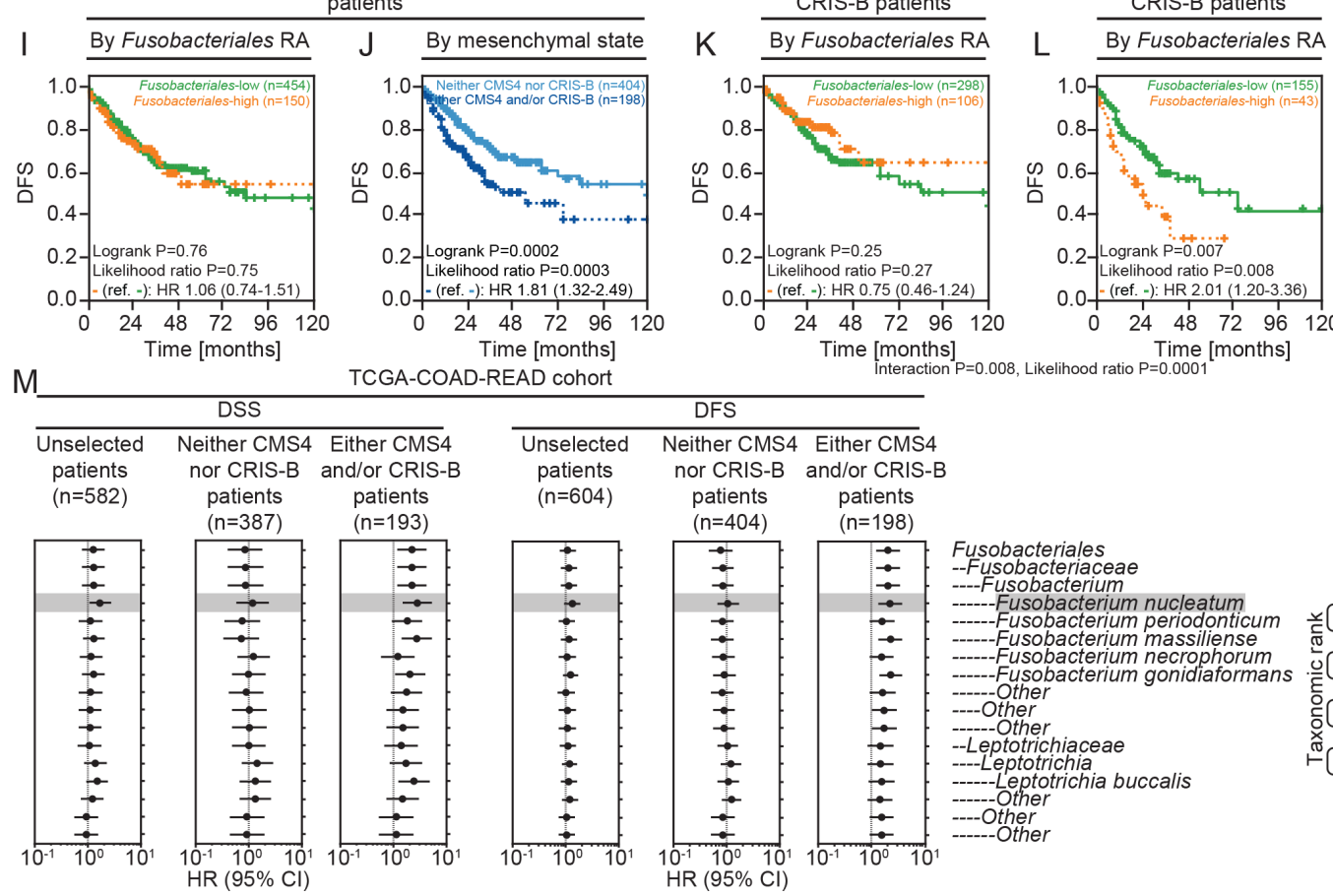

\section{DFS}

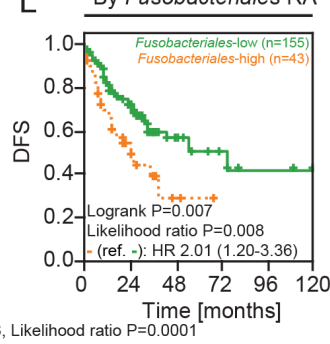

Neither CMS4 Either CMS4

nor CRIS-B and/or CRIS-B

patients patients

$(n=404) \quad(n=198)$
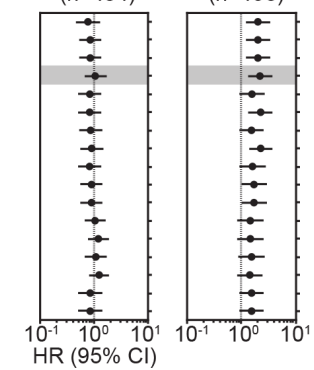

Fusobacteriales
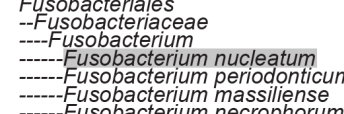

------Fusobacterium necrophorum

-----Other

-----Other

---Leptotrichiace

-----Oeptotrichia buccalis

$\operatorname{HR}(95 \% \mathrm{Cl})$

$$
\begin{aligned}
& \text {-----other } \\
& ----O t h e r \\
& ----O t h e r
\end{aligned}
$$

Figure 5 High Fn/Fusobacteriales prevalence is associated with negative clinical outcome in patients with mesenchymal-like tumours. (A-L) KaplanMeier estimates comparing survival curves in patients of the Taxonomy (OS, A-D) and TCGA-COAD-READ (DSS and DFS cohorts, E-L). Patients across the whole cohort were grouped by bacterium subgroup (low, in green, vs high, in orange; $A, E, I$ ) or mesenchymal status (CMS4 and/or CRIS-B, in light blue, vs remaining cases, in dark blue; $B, F, J)$. Patients were grouped by bacterium group and further stratified by mesenchymal status $(C, D, G, H, K, L)$. Patients were binned into a bacterium group (low vs high) using the $75^{\text {th }}$ percentile as cut-off and based on either Fn load (Taxonomy cohort; A, C-D) or Fusobacteriales RA at the order level (TCGA-COAD-READ cohort; $E, G-I, K, L)$. (M) Cox regression models fitted on bacterium RA reported at the order, family, genus and species taxonomic ranks. for each taxonomic rank, patients were classified as low or high subgroup using the corresponding $75^{\text {th }}$ percentile RA abundance as cut-off. Univariate Cox regression models were fitted when evaluating the association between bacterium subgroup (high vs low, reference low) at each taxonomic rank and either DSS or DFS in the whole unselected patient population (left panel). Cox regression models with an interaction term between bacterium subgroup (high vs low; reference low) and mesenchymal status (mesenchymal, ie, either CMS4 and/or CRIS-B, vs non-mesenchymal, ie, neither CMS4 nor CRIS-B) at each taxonomic rank and either DSS or DFS was fitted to evaluate differential impact of bacterium on clinical outcome by tumour biology (right panels). CMS, consensus molecular subtyping; CRIS, colorectal cancer intrinsic subtyping; DFS, disease-free survival; DSS, disease-specific survival; Fn, Fusobacterium nucleatum; OS, overall survival; TCGA-COAD-READ, colon and rectal cases of The Cancer Genome Atlas. 


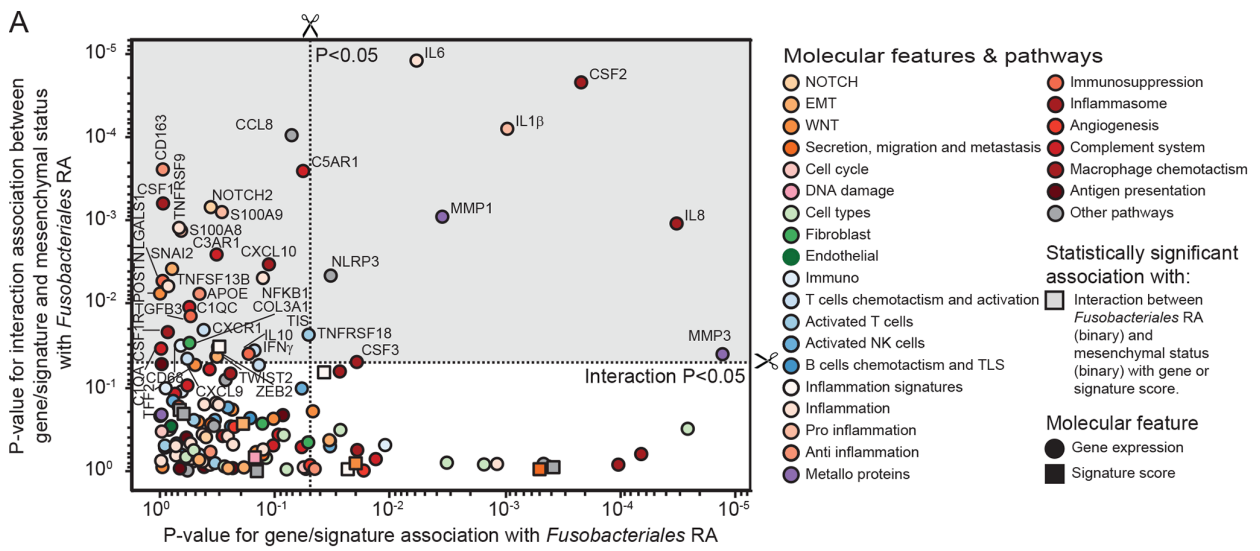

B

Molecular features and signalling pathways

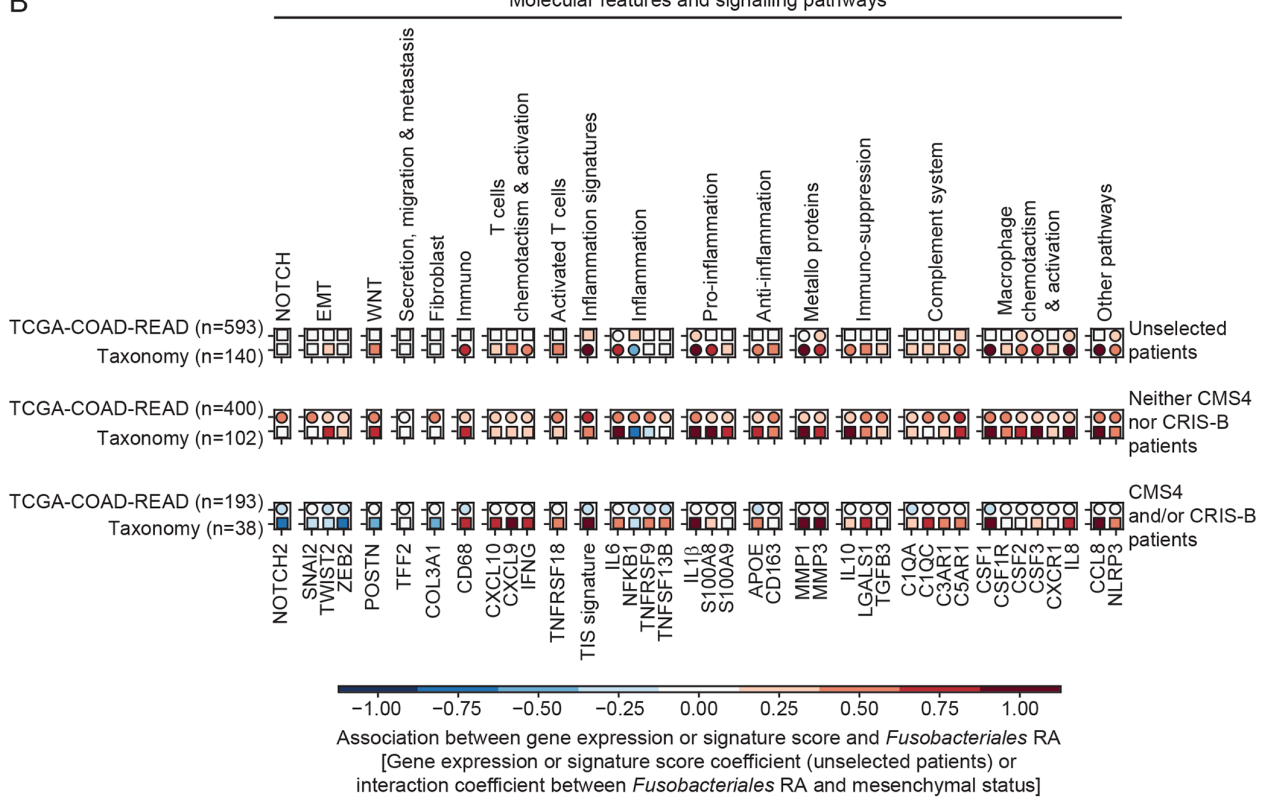

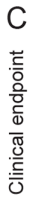

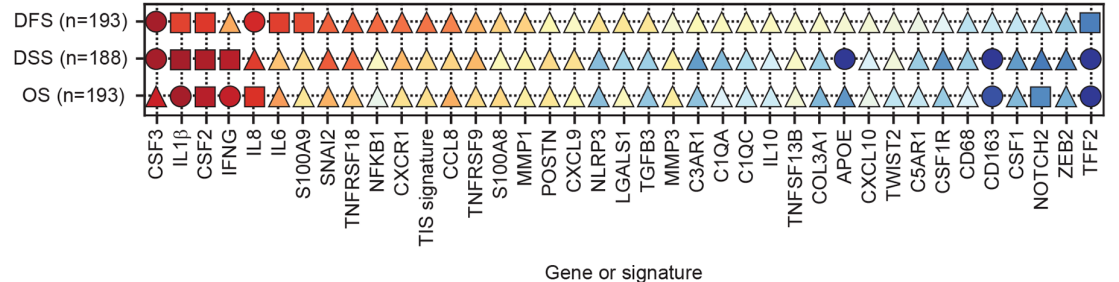

Gene or signature

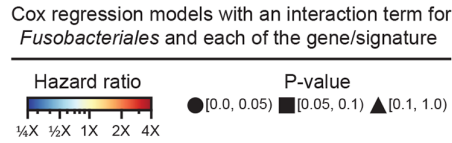

Figure 6 Exploration of mechanism underlying differential impact of Fn/Fusobacteriales in mesenchymal versus non-mesenchymal tumours. (A) Scatterplot depicting $\mathrm{p}$ values derived by assessing with logistic regression models the relationship between genes/signatures associated with Fusobacteriales RA in univariate analysis (model 1, x-axis) or the interaction with mesenchymal status (model 2, y-axis). Gene/signature with statistically significant $p$ values from model 2 are highlighted by a grey shaded area. (B) Breakdown of association including direction and effect size, in the unselected patients' population and within mesenchymal versus non-mesenchymal cases. Only gene/signatures with significant interaction between Fusobacteriales RA and the gene/signature interaction with the molecular subtype (model 2, top quadrant, grey-shaded area) in the TCGACOAD-READ cohort are included. Associations for both the TCGA-COAD-READ (Fusobacteriales RA) and Taxonomy (Fn load) cohorts are shown. Statistically significant associations are represented with circle markers, whereas non-significant associations are indicated by squared markers. (C) Association between gene/signature identified as candidate targets, A) and clinical outcome in patients of the TCGA-COAD-READ cohort with mesenchymal tumours. HRs and $p$ values are derived from Cox regression models with an interaction term for Fusobacteriales relative abundance (low vs high) and each of the gene/signature (low vs high) being evaluated. CMS, consensus molecular subtyping; CRIS, colorectal cancer intrinsic subtyping; DFS, disease-free survival; DSS, disease-specific survival; Fn, Fusobacterium nucleatum; OS, overall survival; TCGA-COAD-READ, colon and rectal cases of The Cancer Genome Atlas. 
Fn/Fusobacteriales has a specific clinical impact in mesenchymalrich, high-stromal infiltrated tumours; this argues against a blanket approach for treating patients with Fn/Fusobacterialeshigh tumours. Treatment with wide spectrum antibiotics reduces the growth of $F n$-positive tumours in vivo. ${ }^{8}$ However, the use of antibiotics to treat $F n$-positive CRC tumours may be limited as $F n$ penetrate deeply within tumour, immune and endothelial cells where they internalise with endosomes and lysosomes, ${ }^{28}$ adapt $^{29}$ and persist. ${ }^{8}$ In addition, long-term use of antibiotics can cause gut dysbiosis, which may impact disease progression and outcome.

Given that 'it takes two to tango', namely, a high pathogen prevalence and a conducive host milieu, we further examined this interdependence to identify druggable aberrations in the host signalling pathways and microenvironment. We identified putative targets related to (pro-)inflammation, inflammasome, activated $\mathrm{T}$ cells, complement system, metalloproteins and macrophage chemotaxis and activation. Fusobacteriales induce a constitutively activated NFKBTNF- $\alpha-$ IL- 6 state which results in activation of metalloproteins and inflammatory cytokines (CSF1-3) which mediate macrophage differentiation, inhibit cytotoxic immune cells and promote proliferation of myeloid-derived-suppressor (MDSC) cells. We observed an increase in inflammation and M1 macrophages and a decrease in M2 macrophages in patients with higher Fn/Fusobacteriales prevalence. We envisage that therapeutic options, such as NLRP3/AIM2 inflammasome suppression, ${ }^{30}$ IL-1 $\beta$ blockade, ${ }^{31}$ TNF- $\alpha{ }^{32}$ or IL-6 inhibition, ${ }^{33}$ which have been approved for treatment of chronic inflammation and cytokine storm syndrome in multiple cancers, rheumatoid arthritis and COVID-19 may ameliorate the immunosuppressive microenvironment

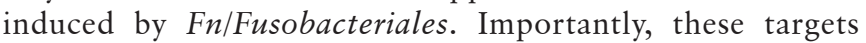
are involved in not only promoting an immunosuppressive microenvironment by recruiting tissue-associated macrophages (TAMs) and MDSCs, but also in orchestrating invasion, angiogenesis, epithelial-to-mesenchymal transition and, ultimately, metastasis. The prometastatic impact of Fn/Fusobacteriales is further corroborated by findings in the literature linking higher pathogen prevalence in more advanced disease stage and metastasis in clinical specimens ${ }^{5}$ and higher metastatic burden in mice inoculated with $F n .{ }^{34}$

Cancer cells with an EMT phenotype secrete cytokines such as IL-10 and TGF- $\beta$ that can further promote an immunosuppressive microenvironment. Additionally, secretion of IL-6 and IL-8 from stroma cells can further foster an EMT phenotype, activate primary fibroblasts (cancer-associated fibroblast (CAFs)) which, in turn, may promote angiogenesis and invasion. ${ }^{35}$ Taken together, these aberrations may result in a self-reinforcing mechanism that confers on cancer cells the ability to migrate, invade the extracellular matrix, extravasate and seed metastasis. When comparing the transcriptomic profiles by Fusobacteriales RA in the TCGA-COAD-READ cohort, we identified dysregulation affecting cell architecture involving apical surface dynamics and Aurora A kinase signalling, which regulate cMYC, DNA repair, cell motility/migration and induce EMT transition via $\beta$-catenin and TGF- $\beta$, leading to metastasis and resistance to treatment in multiple cancer types. ${ }^{36}$ Small molecule inhibitors against Aurora A have shown encouraging results in preclinical studies and clinical trials in $\mathrm{CRC}^{37}$ and other cancers. ${ }^{38}$ Cytoskeleton shape, filopodium protrusions and alterations in cell adhesion and structure are hallmarks of extracellular matrix invasion. EMT key effectors,
SNAIL and ZEB1, alter apical surface dynamics by inhibiting scaffolding proteins and by inducing expression of matrix metalloproteins (MMP3 and MMP9), resulting in loosened tight junctions, altered cell polarity and increased plasticity which, in turn, enable cell invasion. ${ }^{39}$ Dysregulations in MMP expression may aid cancer cells that have reached the bloodstream to extravasate to distant tissues ${ }^{40}$ by priming the vascular endothelium via upregulation of VEGF- $\mathrm{A}^{41}$ and by increasing permeability via COX2 upregulation. ${ }^{42}$ Our analyses in the TCGA-COAD-READ cohort identified higher expression of vascular endothelial growth factor (VEGF) as well as an angiogenesis signature in patients with higher Fusobacteriales RA. Indeed, a new generation of selective and highly penetrative MMP inhibitors ${ }^{43}$ is being trialled in GI cancers, ${ }^{44}$ and Mehta et al reported lower Fusobacteriales $\mathrm{RA}$ in subjects treated with aspirin, a COX2 inhibitor. ${ }^{45}$

Green et $a l^{46}$ demonstrated that MAPK7 is a master regulator of MMP9 and promotes the formation of metastasis. We observed a dysregulation in MAPK signalling at the protein level when comparing Fusobacteriales-high versus Fusobacteriales-low patients of the TCGA-COAD-READ cohort. MAPK7 induces EMT transition, cell migration and regulates TAM polarisation in a metalloprotein-dependent manner ${ }^{46}$ rendering it an appealing upstream therapeutic target. IL-6 orchestrates MAPK-STAT3 signalling, which in turn regulates the dynamic transition between two CAFs subpopulations, EMT-CAFs and proliferation-CAFs, ${ }^{47}$ rendering the IL-6-TGF- $\beta$-EMT-CAFs cross-talk potentially a further therapeutic target. While directly targeting EMT via NOTCH or WNT has shown limited success in the clinic, ${ }^{48}$ microenvironment remodelling to reverse immunosuppression by inhibiting CXCL12 ${ }^{49}$ or promoting T-cell infiltra$\operatorname{tion}^{50}$ or function via engineered oncolytic adenovirus, ${ }^{51}$ has shown promising results in reducing metastasis formation. ${ }^{52}$ Additionally, we observed a positive correlation between gene expression of IL-8, CXCL8, CXCR1 and CXCL10 and $\mathrm{Fn} /$ Fusobacteriales prevalence, corroborating findings from Casasanta et al. assessing Fn in HCT116 CRC cells. ${ }^{53}$

In conclusion, our analyses have identified a patient subpopulation that has an unfavourable clinical outcome when their tumours exhibit mesenchymal traits and are highly positive with Fn/Fusobacteriales and pinpointed clinically actionable hostspecific vulnerabilities that suggest new treatments for these patients that extend beyond broad-spectrum antibiotics.

Acknowledgements We thank the patients who kindly donated their samples and made this study possible.

Contributors MS, DL and JHMP conceptualised and designed the study. MS, $N C, K S, S B, D L$ and JHMP were involved in collection, preparation, interpretation, validation and critical review of the data. NC and KS performed the cell culture experiments and quantified bacterium load in tumour samples of the patients of the Taxonomy cohort. MS performed formal analysis including bioinformatics and statistical analyses. MS created the manuscript figures and supplementary materials. MS and JHMP drafted the manuscript. All authors edited, reviewed, revised and approved the manuscript text. DL and JHMP acquired funding for the study.

Funding This study was supported by a grant from the Health Research Board and Science Foundation Ireland to JHMP (16/US/3301), a studentship to KS sponsored by The Northern Ireland Department for the Economy (NI DfE), and by funding from NI DFE (SFI-DEL 14/1A/2582, STL/5715/15). The results included here are in part based on data generated by the TCGA Research Network (https://wwwcancergov/tcga). We wish to acknowledge the information technology department at the Royal College of Surgeons in Ireland and the Irish Centre for High-End Computing (ICHEC) for the provision of computational facilities and support.

Competing interests None declared.

Patient consent for publication Not required. 
Ethics approval The Taxonomy cohort collection was approved by the Medicine, Dentistry, and Biomedical Sciences School Ethics Committee (ref: 12/12v4), as previously described (Allen et al, JCO Precision Oncology, 2018; PMID: 30088816). Approval for the cohort of patients with colon (COAD) and rectal (READ) cases denotated as TCGA-COAD-READ in this manuscript was acquired by the original investigators of The Cancer Genome Atlas consortium.

Provenance and peer review Not commissioned; externally peer reviewed.

Data availability statement Data are available in a public, open access repository. Processing and analysis code along with bacterium estimates with corresponding clinical and molecular datasets for the Taxonomy and TCGA-COADREAD cohorts included in this study are publicly available and archived at Zenodo (https://10.5281/zenodo.4019142). Bacterium estimates include Fusobacterium nucleatum load (Taxonomy cohort) and Fusobacteriales relative abundance, along with higher resolution estimates at genus, family and species taxonomic ranks, (TCGA-COAD-READ cohort).

Supplemental material This content has been supplied by the author(s). It has not been vetted by BMJ Publishing Group Limited (BMJ) and may not have been peer-reviewed. Any opinions or recommendations discussed are solely those of the author(s) and are not endorsed by BMJ. BMJ disclaims all liability and responsibility arising from any reliance placed on the content. Where the content includes any translated material, BMJ does not warrant the accuracy and reliability of the translations (including but not limited to local regulations, clinical guidelines, terminology, drug names and drug dosages), and is not responsible for any error and/or omissions arising from translation and adaptation or otherwise.

Open access This is an open access article distributed in accordance with the Creative Commons Attribution Non Commercial (CC BY-NC 4.0) license, which permits others to distribute, remix, adapt, build upon this work non-commercially, and license their derivative works on different terms, provided the original work is properly cited, appropriate credit is given, any changes made indicated, and the use is non-commercial. See: http://creativecommons.org/licenses/by-nc/4.0/.

\section{ORCID iD}

Manuela Salvucci http://orcid.org/0000-0001-9941-4307

\section{REFERENCES}

1 Bray F, Ferlay J, Soerjomataram I, et al. Global cancer statistics 2018: GLOBOCAN estimates of incidence and mortality worldwide for 36 cancers in 185 countries. CA Cancer I Clin 2018:68:394-424.

2 Guinney J, Dienstmann R, Wang X, et al. The consensus molecular subtypes of colorectal cancer. Nat Med 2015;21:1350-6.

3 Isella C, Brundu F, Bellomo SE, et al. Selective analysis of cancer-cell intrinsic transcriptional traits defines novel clinically relevant subtypes of colorectal cancer. Nat Commun 2017;8:15107.

4 Routy B, Gopalakrishnan V, Daillère $R$, et al. The gut microbiota influences anticancer immunosurveillance and general health. Nat Rev Clin Oncol 2018;15:382-96.

5 Flanagan L, Schmid J, Ebert M, et al. Fusobacterium nucleatum associates with stages of colorectal neoplasia development, colorectal cancer and disease outcome. Eur J Clin Microbiol Infect Dis 2014;33:1381-90.

6 Brennan CA, Garrett WS. Fusobacterium nucleatum - symbiont, opportunist and oncobacterium. Nat Rev Microbiol 2019;17:156-66.

7 Ito M, Kanno S, Nosho K, et al. Association of Fusobacterium nucleatum with clinical and molecular features in colorectal serrated pathway. Int I Cancer 2015;137:1258-68.

8 Bullman S, Pedamallu CS, Sicinska E, et al. Analysis of Fusobacterium persistence and antibiotic response in colorectal cancer. Science 2017;358:1443-8.

9 Gethings-Behncke C, Coleman HG, Jordao HWT, et al. Fusobacterium nucleatum in the Colorectum and Its Association with Cancer Risk and Survival: A Systematic Review and Meta-analysis. Cancer Epidemiol Biomarkers Prev 2020;29:539-48.

10 Dharmani P, Strauss J, Ambrose C, et al. Fusobacterium nucleatum infection of colonic cells stimulates MUC2 mucin and tumor necrosis factor alpha. Infect Immun 2011;79:2597-607.

11 Kostic AD, Chun E, Robertson L, et al. Fusobacterium nucleatum potentiates intestinal tumorigenesis and modulates the tumor-immune microenvironment. Cell Host Microbe 2013;14:207-15.

12 Allen WL, Dunne PD, McDade S, et al. Transcriptional subtyping and CD8 immunohistochemistry identifies patients with stage II and III colorectal cancer with poor prognosis who benefit from adjuvant chemotherapy. JCO Precis Oncol 2018;21:1-15.

13 McCorry AM, Loughrey MB, Longley DB, et al. Epithelial-To-Mesenchymal transition signature assessment in colorectal cancer quantifies tumour stromal content rather than true transition. J Pathol 2018;246:422-6.

14 Kostic AD, Ojesina Al, Pedamallu CS, et al. PathSeq: software to identify or discover microbes by deep sequencing of human tissue. Nat Biotechnol 2011;29:393-6.
15 Walker MA, Pedamallu CS, Ojesina Al, et al. GATK PathSeq: a customizable computational tool for the discovery and identification of microbial sequences in libraries from eukaryotic hosts. Bioinformatics 2018;215:4287-9.

16 Finotello F, Mayer C, Plattner C, et al. Molecular and pharmacological modulators of the tumor immune contexture revealed by deconvolution of RNA-Seq data. Genome Med 2019;11:34.

17 Becht E, Giraldo NA, Lacroix L, et al. Estimating the population abundance of tissueinfiltrating immune and stromal cell populations using gene expression. Genome Biol 2016;17:218.

18 Mima K, Sukawa Y, Nishihara R, et al. Fusobacterium nucleatum and T Cells in Colorectal Carcinoma. JAMA Oncol 2015;1:653-61.

19 Mermel CH, Schumacher SE, Hill B, et al. GISTIC2.0 facilitates sensitive and confident localization of the targets of focal somatic copy-number alteration in human cancers. Genome Biol 2011;12:R41.

20 Castellarin M, Warren RL, Freeman JD, et al. Fusobacterium nucleatum infection is prevalent in human colorectal carcinoma. Genome Res 2012;22:299-306.

21 McCoy AN, Araújo-Pérez F, Azcárate-Peril A, et al. Fusobacterium is associated with colorectal adenomas. PLoS One 2013;8:e53653.

22 Kostic AD, Gevers D, Pedamallu CS, et al. Genomic analysis identifies association of Fusobacterium with colorectal carcinoma. Genome Res 2012;22:292-8.

23 Nosho K, Sukawa Y, Adachi Y, et al. Association of Fusobacterium nucleatum with immunity and molecular alterations in colorectal cancer. World J Gastroenterol 2016;22:557-66.

24 Kinross J, Mirnezami R, Alexander J, et al. A prospective analysis of mucosal microbiome-metabonome interactions in colorectal cancer using a combined MAS 1HNMR and metataxonomic strategy. Sci Rep 2017;7:8979.

25 Lee D-W, Han S-W, Kang J-K, et al. Association between Fusobacterium nucleatum, pathway mutation, and patient prognosis in colorectal cancer. Ann Surg Oncol 2018;25:3389-95.

26 Kunzmann AT, Proença MA, Jordao HW, et al. Fusobacterium nucleatum tumor DNA levels are associated with survival in colorectal cancer patients. Eur J Clin Microbiol Infect Dis 2019;38:1891-9.

27 Purcell RV, Visnovska M, Biggs PJ, et al. Distinct gut microbiome patterns associate with consensus molecular subtypes of colorectal cancer. Sci Rep 2017;7:11590

$28 \mathrm{Ji} \mathrm{S}$, Shin JE, Kim YC, et al. Intracellular degradation of Fusobacterium nucleatum in human gingival epithelial cells. Mol Cells 2010;30:519-26.

29 Umaña A, Sanders BE, Yoo CC, et al. Utilizing Whole Fusobacterium Genomes To Identify, Correct, and Characterize Potential Virulence Protein Families. J Bacteriol 2019:201:e00273-19.

30 Hamarsheh Shaima'a, Zeiser R. Nlrp3 inflammasome activation in cancer: a doubleedged sword. Front Immunol 2020;11.

31 Dinarello CA, Simon A, van der Meer JWM. Treating inflammation by blocking interleukin-1 in a broad spectrum of diseases. Nat Rev Drug Discov 2012;11:633-52.

32 Ortiz P, Bissada NF, Palomo L, et al. Periodontal therapy reduces the severity of active rheumatoid arthritis in patients treated with or without tumor necrosis factor inhibitors. J Periodontol 2009;80:535-40.

33 Choy EH, De Benedetti F, Takeuchi T, et al. Translating IL-6 biology into effective treatments. Nat Rev Rheumatol 2020;16:335-45.

34 Parhi L, Alon-Maimon T, Sol A, et al. Breast cancer colonization by Fusobacterium nucleatum accelerates tumor growth and metastatic progression. Nat Commun 2020;11:1-12

35 Erez N, Truitt M, Olson P, et al. Cancer-Associated fibroblasts are activated in incipient neoplasia to orchestrate tumor-promoting inflammation in an NF- $\mathrm{KB}$-dependent manner. Cancer Cell 2010;17:135-47.

36 Shah K, Ahmed M, Kazi JU. The Aurora kinase//-catenin axis contributes to dexamethasone resistance in leukemia. NPJ Precis Oncol 2021;5:13.

37 Pitts TM, Bradshaw-Pierce EL, Bagby SM, et al. Antitumor activity of the Aurora a selective kinase inhibitor, alisertib, against preclinical models of colorectal cancer. Oncotarget 2016;7:50290-301.

38 Brockmann M, Poon E, Berry T, et al. Small molecule inhibitors of Aurora-A induce proteasomal degradation of $\mathrm{N}$-myc in childhood neuroblastoma. Cancer Cell 2013;24:75-89.

39 Stemmler MP, Eccles RL, Brabletz S, et al. Non-Redundant functions of EMT transcription factors. Nat Cell Biol 2019;21:102-12.

40 Cox TR. The matrix in cancer. Nat Rev Cancer 2021:21:217-38.

41 Desch A, Strozyk EA, Bauer AT, et al. Highly invasive melanoma cells activate the vascular endothelium via an MMP-2/integrin $\alpha v \beta 5$-induced secretion of VEGF-A. Am J Pathol 2012;181:693-705.

42 Lee KY, Kim Y-J, Yoo H, et al. Human brain endothelial cell-derived COX-2 facilitates extravasation of breast cancer cells across the blood-brain barrier. Anticancer Res 2011;31:4307-13.

43 Winer A, Adams S, Mignatti P. Matrix metalloproteinase inhibitors in cancer therapy: turning past failures into future successes. Mol Cancer Ther 2018;17:1147-55.

44 Bendell JC, Starodub A, Huang X, et al. A phase 3 randomized, double-blind, placebo-controlled study to evaluate the efficacy and safety of GS-5745 combined with mFOLFOX6 as first-line treatment in patients with advanced gastric or gastroesophageal junction adenocarcinoma. JCO 2017;35:TPS4139. 
45 Mehta RS, Nishihara R, Cao Y, et al. Association of dietary patterns with risk of colorectal cancer subtypes classified by Fusobacterium nucleatum in tumor tissue. JAMA Oncol 2017;3:921.

46 Green D, Eyre $H$, Singh A, et al. Targeting the MAPK7/MMP9 axis for metastasis in primary bone cancer. Oncogene 2020;39:5553-69.

47 Ligorio M, Sil S, Malagon-Lopez J, et al. Stromal microenvironment shapes the intratumoral architecture of pancreatic cancer. Cell 2019;178:160-75.

48 Strosberg JR, Yeatman T, Weber J, et al. A phase II study of ro4929097 in metastatic colorectal cancer. Eur J Cancer 2012;48:997-1003.

49 Lang J, Zhao X, Qi Y, et al. Reshaping Prostate Tumor Microenvironment To Suppress Metastasis via Cancer-Associated Fibroblast Inactivation with Peptide-AssemblyBased Nanosystem. ACS Nano 2019;13:12357-71.
50 Tauriello DVF, Palomo-Ponce $S$, Stork D, et al. Tgf $\beta$ drives immune evasion in genetically reconstituted colon cancer metastasis. Nature 2018;554:538-43.

51 Freedman JD, Duffy MR, Lei-Rossmann J, et al. An oncolytic virus expressing a T-cell Engager simultaneously targets cancer and immunosuppressive stromal cells. Cancer Res 2018;78:6852-65.

52 Ping Q, Yan R, Cheng X, et al. Correction: cancer-associated fibroblasts: overview, progress, challenges, and directions. Cancer Gene Ther 2021. doi:10.1038/s41417021-00343-3. [Epub ahead of print: 28 Jun 2021].

53 Casasanta MA, Yoo CC, Udayasuryan B, et al. Fusobacterium nucleatum host-cell binding and invasion induces IL-8 and CXCL1 secretion that drives colorectal cancer cell migration. Sci Signal 2020;13:eaba9157. 